\title{
Magnetic geometries of Sun-like stars: exploring the mass-rotation plane
}

\author{
Pascal Petit $^{1}$, B. Dintrans ${ }^{1}$, M. Aurière ${ }^{1}$, C. Catala ${ }^{2}$, J.-F. Donati ${ }^{1}$, \\ R. Fares ${ }^{1}$, T. Gastine ${ }^{1}$, F. Lignières ${ }^{1}$, A. Morgenthaler ${ }^{1}$, J. Morin ${ }^{1}$, \\ F. Paletou ${ }^{1}$, J. Ramirez ${ }^{2}$, S.K. Solanki ${ }^{3}$, and S. Théado ${ }^{1}$ \\ ${ }^{1}$ Laboratoire d'Astrophysique de Toulouse-Tarbes, Universit de Toulouse, CNRS, France \\ email: petit@ast.obs-mip.fr, dintrans@ast.obs-mip.fr, auriere@ast.obs-mip.fr, \\ donati@ast.obs-mip.fr, rim.fares@ast.obs-mip.fr, thomas.gastine@ast.obs-mip.fr, \\ francois.lignieres@ast.obs-mip.fr, amorgent@ast.obs-mip.fr, julien.morin@ast.obs-mip.fr, \\ fpaletou@ast.obs-mip.fr, stheado@ast.obs-mip.fr \\ ${ }^{2}$ LESIA, Observatoire de Paris-Meudon, 92195 Meudon, France \\ email: claude.catala@obspm.fr, julio.ramirez@obspm.fr \\ ${ }^{3}$ Max-Planck-Institut fr Sonnensystemforschung, Max-Planck-Str. 2, 37191 \\ Katlenburg-Lindau, Germany, email: solanki@mps.mpg.de
}

\begin{abstract}
Sun-like stars are able to continuously generate a large-scale magnetic field through the action of a dynamo. Various physical parameters of the star are able to affect the dynamo output, in particular the rotation and mass. Using the NARVAL spectropolarimeter (Observatoire du Pic du Midi, France), it is now possible to measure the large-scale magnetic field of solar analogues (i.e. stars very close to the Sun in the mass-rotation plane, including strict solar twins). From spectropolarimetric time-series, tomographic inversion enables one to reconstruct the field geometry and its progressive distortion under the effect of surface differential rotation. We show the first results obtained on a sample of main-sequence dwarfs, probing masses between 0.7 and 1.4 solar mass and rotation rates between 1 and 3 solar rotation rate.
\end{abstract}

Keywords. Stars: activity - stars: atmospheres - stars: imaging - stars: late-type - stars: magnetic fields - stars: rotation

Spectropolarimetric observations of a sample of late-type main-sequence dwarfs have been obtained with the NARVAL spectropolarimeter (Petit et al. 2008). The full stellar sample at our disposal is now constituted of about 20 objects probing a range of stellar masses and rotation rates.

A single, average photospheric line profile was extracted from each spectrum using the LSD technique (Donati et al. 1997), according to line-lists matching various stellar photospheric models calculated for the spectral types of our target list. Using this crosscorrelation method, the noise level of the mean Stokes $\mathrm{V}$ profiles is reduced by a factor of about 40 with respect to the initial spectrum. The resulting noise levels are in the range $2 \times 10^{-5}-10^{-4} I_{\mathrm{c}}$ (where $I_{\mathrm{c}}$ denotes the intensity of continuum), depending on the stellar magnitude, spectral type, exposure time and observing conditions.

Assuming that the observed temporal variability of Stokes V profiles is controlled by the stellar rotation (including a possible latitudinal shear, Petit et al. 2002), we reconstruct the magnetic geometry of our targets by means of Zeeman-Doppler Imaging. We employ here the modelling approach of Donati \& Brown (1997), including also the spherical harmonics expansion of the surface magnetic field implemented by Donati et al. (2006) in order to easily distinguish between the poloidal and toroidal components of the reconstructed magnetic field distribution. 
radial magnetic field $(G)$

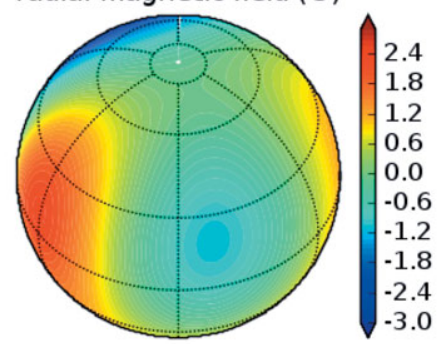

meridional magnetic field (G)

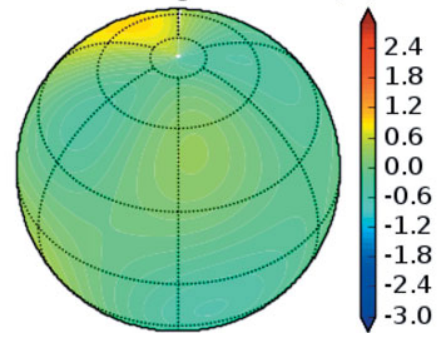

azimuthal magnetic field (G)

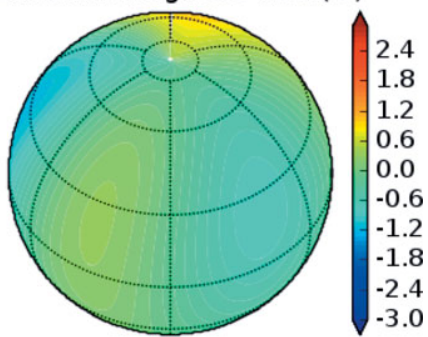

Figure 1. Magnetic map of the solar twin 18 Sco. Each chart illustrates the field projection onto one axis of the spherical coordinate frame. The magnetic field strength is expressed in Gauss.

From the set of reconstructed magnetic maps, we find that stars with high Rossby numbers (defined as $P_{\text {rot }} / \tau_{c}$, where $P_{\text {rot }}$ is the rotation period and $\tau_{c}$ the convective turnover time) feature weak, mostly poloidal surface fields. Stars with smaller Rossby numbers are able to generate stronger magnetic fields, with a mainly toroidal field geometry at photospheric level.

We plan to monitor the selected targets over 5 to 10 years to estimate the longterm variability of their magnetic topologies and the dependence of magnetic cycles on various stellar parameters. The temporal evolution of the total magnetic energy, the poloidal/toroidal distribution of the surface field or the distribution of the magnetic energy between the axisymmetric and non-axisymmetric components will then provide us with a new set of surface observables that will help to constrain numerical models of stellar dynamos.

\section{References}

Donati, J.-F., et al. 1997, MNRAS 291, 658

Donati J.-F. \& Brown S. F., 1997, A\&A 326, 1135

Donati, J.-F., et al. 2006, MNRAS 370, 629

Petit, P., et al. 2002, MNRAS 334, 374

Petit, P., et al. 2008, MNRAS 388, 80

Sanderson, T. R., et al. 2003, JGR 108, SSH 7-1

Wright, J. T., et al. 2004, ApJS 152, 261 